\title{
Surgical management of pilon fractures with large segmental bone defects using fibular strut allografts: a report of two cases
}

\author{
Weon-Yoo Kim $\cdot$ Jong-Hun Ji $\cdot$ Sang-Eun Park $\cdot$ \\ Young-Yul Kim $\cdot$ Jae Jung Jeong $\cdot$ Hyun-Taek Kang
}

Received: 21 July 2010 / Accepted: 20 November 2010 / Published online: 25 March 2011

(C) The Author(s) 2011. This article is published with open access at Springerlink.com

\begin{abstract}
We present two patients with open pilon fractures with large bone defects treated successfully with fibular strut allografts. The patients were initially treated by massive irrigation, wound debridement, and temporary external fixation. After complete wound healing, the bone defects were managed. Because autologous iliac crest or fibular bone grafts were impossible to be harvested due to multiple fractures, the bone defects were reconstructed with fibular strut allografts. Fixation was performed with a periarticular distal tibia locking plate. At 2 months postoperatively, the patients ambulated with partial weight-bearing; at 6 months, they had full range of motion of the ankle joint and full weight-bearing.
\end{abstract}

Keywords Pilon fracture - Large segmental bone defect . Fibular strut allograft $\cdot$ Locking plate

\section{Introduction}

Several treatment modalities, such as bone grafting, vascularized local or free bone transfer, and distraction osteogenesis, have been recently used for large segmental bone defects of the tibia [1-8]. Although satisfactory results have been reported with these techniques, a limitation of joint motion due to long-term immobilization and non-weight bearing due to weak grafts are problems $[4,6]$. Also, when autogenous iliac bone graft and fibular bone grafts are

W.-Y. Kim · J.-H. Ji · S.-E. Park · Y.-Y. Kim •

J. J. Jeong $(\square) \cdot$ H.-T. Kang

Department of Orthopaedic Surgery,

Daejeon St. Mary's Hospital,

The Catholic University of Korea, Daejeon, South Korea

e-mail: drjj21@hanmail.net impossible due to multiple fractures, there are few treatment options for large segmental bone defect.

We present two patients with open pilon fractures with large bone defects treated successfully with fibular strut allografts. The patients were initially treated by massive irrigation, wound debridement, and temporary external fixation. After complete wound healing, the bone defects were managed. Because autologous iliac crest or fibular bone grafts were impossible to be harvested due to multiple fractures, the bone defects were reconstructed with fibular strut allografts. Fixation was performed with a periarticular distal tibia locking plate. At 2 months postoperatively, the patients ambulated with partial weight-bearing; at 6 months, they had full range of motion of the ankle joint and full weight-bearing.

\section{Case reports}

Case report 1

A 40-year-old woman with schizophrenia attempted suicide by falling from the 4th floor of a building. She had multiple fractures involving the calcaneus bilaterally, spine, and the right distal tibia and fibula. Plain radiographs of the right ankle showed a Gustilo-Anderson type IIIA open pilon fracture with a large segmental bone defect approximately $10 \mathrm{~cm}$ in size at the distal tibia (Fig. 1). Because neurologic symptoms resulted from the spine fracture, the patient had to undergo an emergency spine operation. Massive irrigation and wide debridement of the right leg were performed at the same time. For the length and stability of the right leg, which was shortened by a large segmental bone defect, open reduction and internal fixation (ORIF) in the fibula were performed using a 1/3 tubular plate. And an Ilizarov 


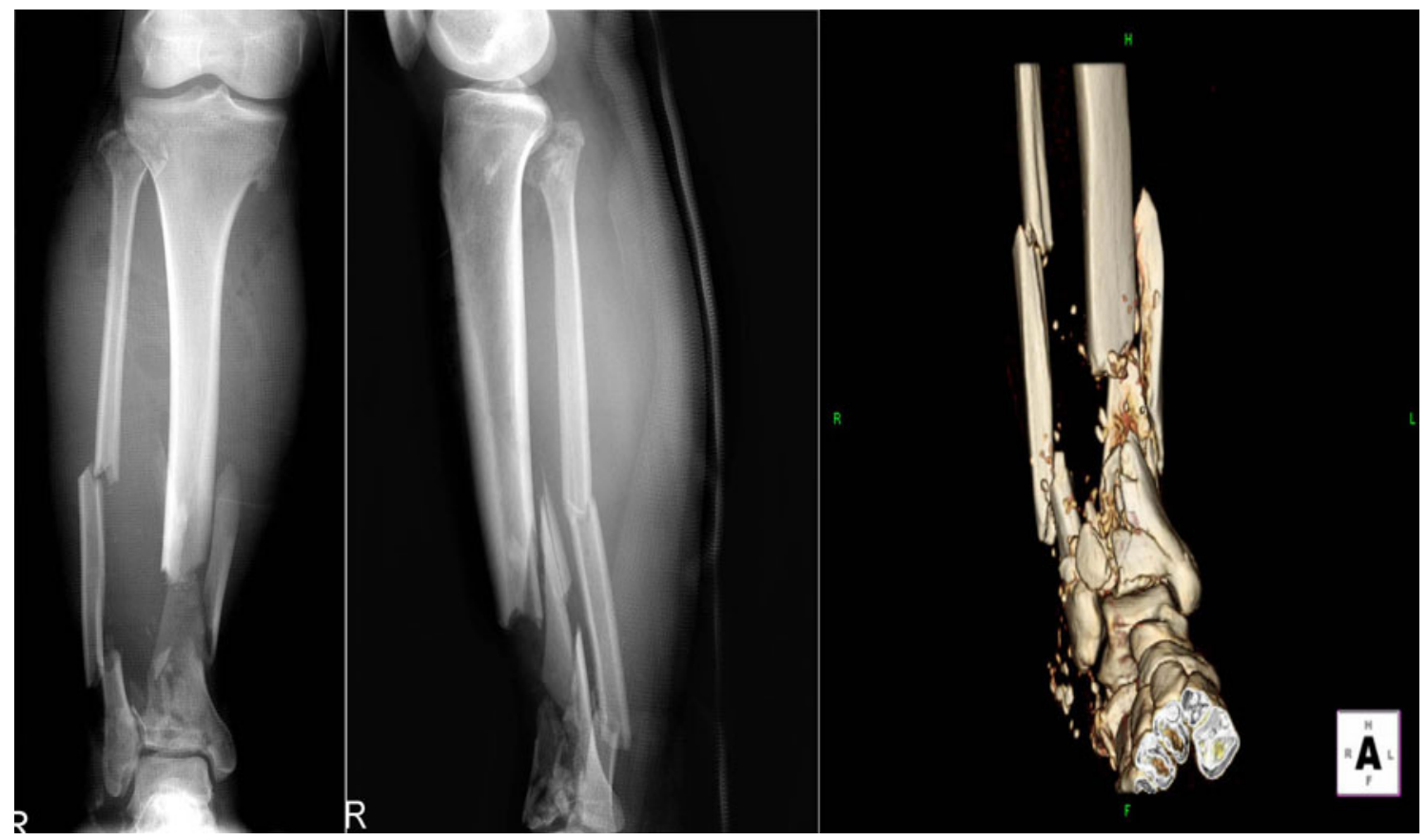

Fig. 1 Plain radiograph and three-dimensional computed tomogram show a highly comminuted pilon fracture with large metaphyseal bone defect

Fig. 2 ORIF of the fracture of the fibula and application of a spanning external fixator

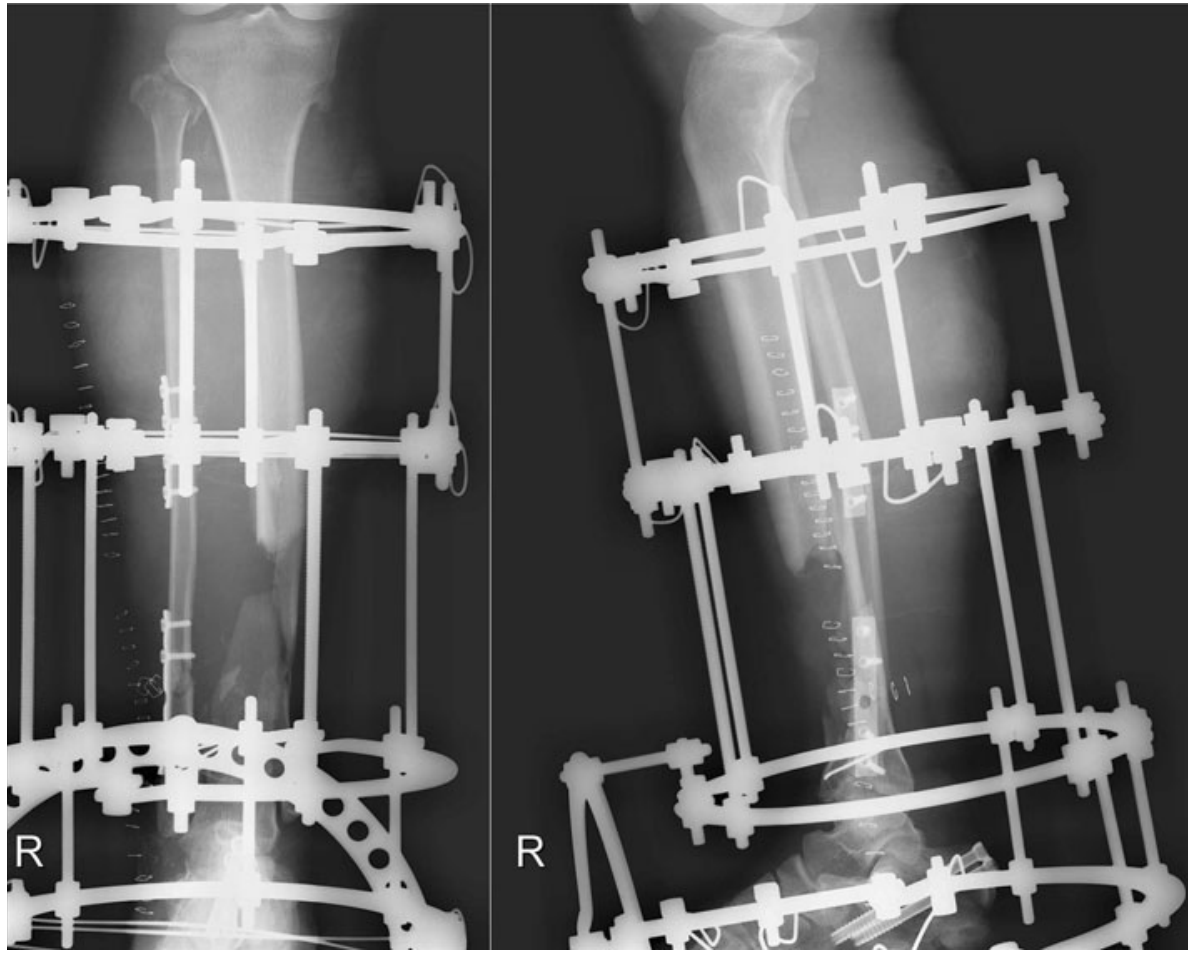

to infection, we delayed the operation after complete wound healing and normalized laboratory findings, such as the erythrocyte sedimentation rate (ESR) and C-reactive protein (CRP) level. Minor debridement was performed under local anesthesia due to mild serous discharge from open wound site. The wound was completely healed after 4 weeks. Then, the external fixator was removed, and we 


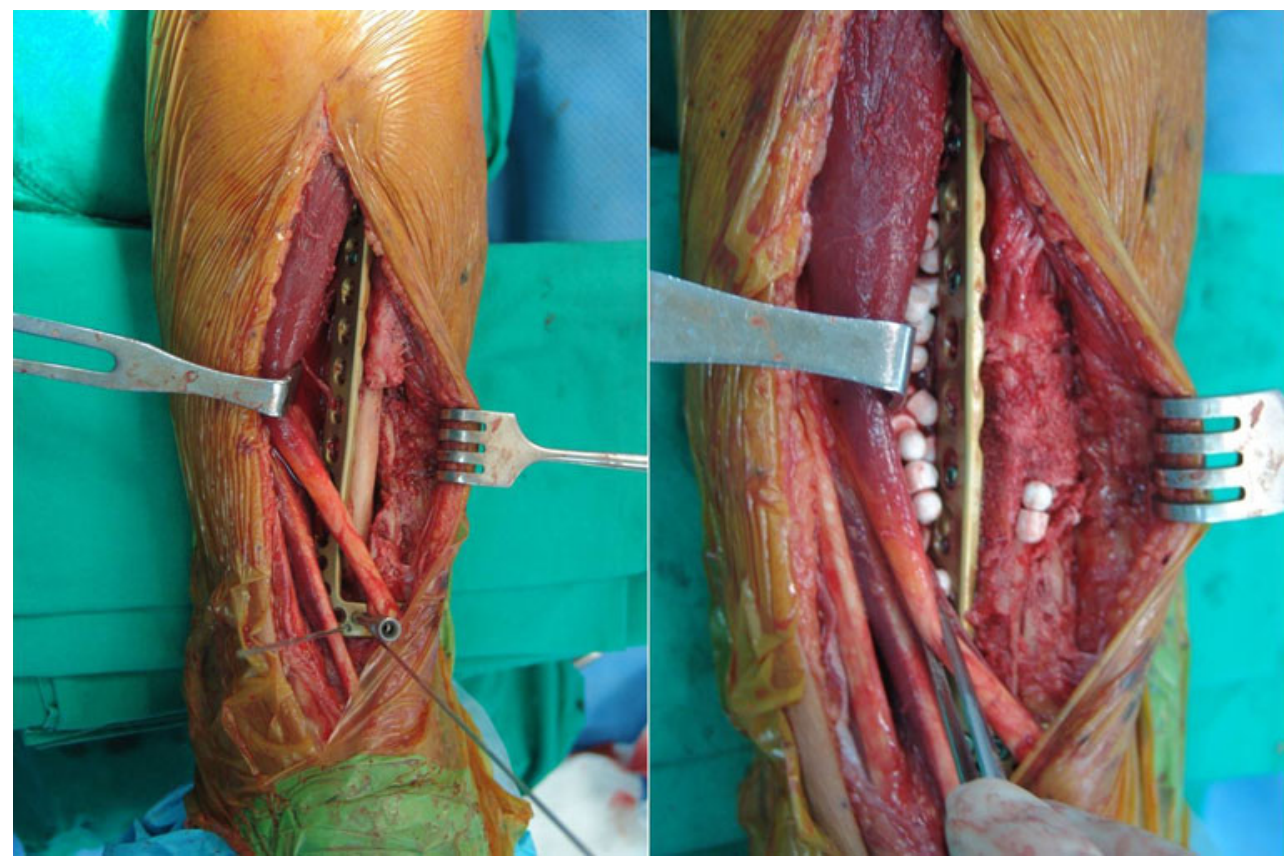

Fig. 3 Intraoperative photograph after reconstruction of the defect using an intramedullary fibular strut allograft and ORIF using a periarticular distal tibia locking plate

performed ORIF using fibular strut allograft. First, the large segmental defect was exposed via an anterior approach. Second, the fibular strut allograft was impacted to the medullary canal of the tibia. Then, the fixation was performed using a periarticular distal tibia locking plate (Synthes ${ }^{\circledR}$, Switzerland). Finally, the iliac auto-bone and calcium sulfate (bone substitute) were added to enhance bone union (Fig. 3). We obtained the normal alignment and rigid fixation postoperatively (Fig. 4).

The patient was encouraged to do active ankle motion with non-weight bearing for accelerating rehabilitation immediately after ORIF. One month later, the patient was discharged without wound problems. From 2 months after ORIF, the patient started partial weight-bearing ambulation. Four months thereafter, the patient could ambulate without any assistance and had a full range of ankle motion. At the 2-year follow-up, the patient ambulated well without specific problems (Fig. 5).

\section{Case report 2}

A 41-year-old woman had a fall injury from the 5th floor. Plain radiographs showed multiple fractures involving the pelvis and sacrum, shaft of femur and humerus, L2 vertebra, and both ankles. Plain radiograph of the left ankle showed a Gustilo-Anderson type IIIA open pilon fracture with a large bone defect approximately $7 \mathrm{~cm}$ in size at the distal tibia (Fig. 6). To manage the multiple fractures, a sacroiliac screw and a pelvic external fixator were

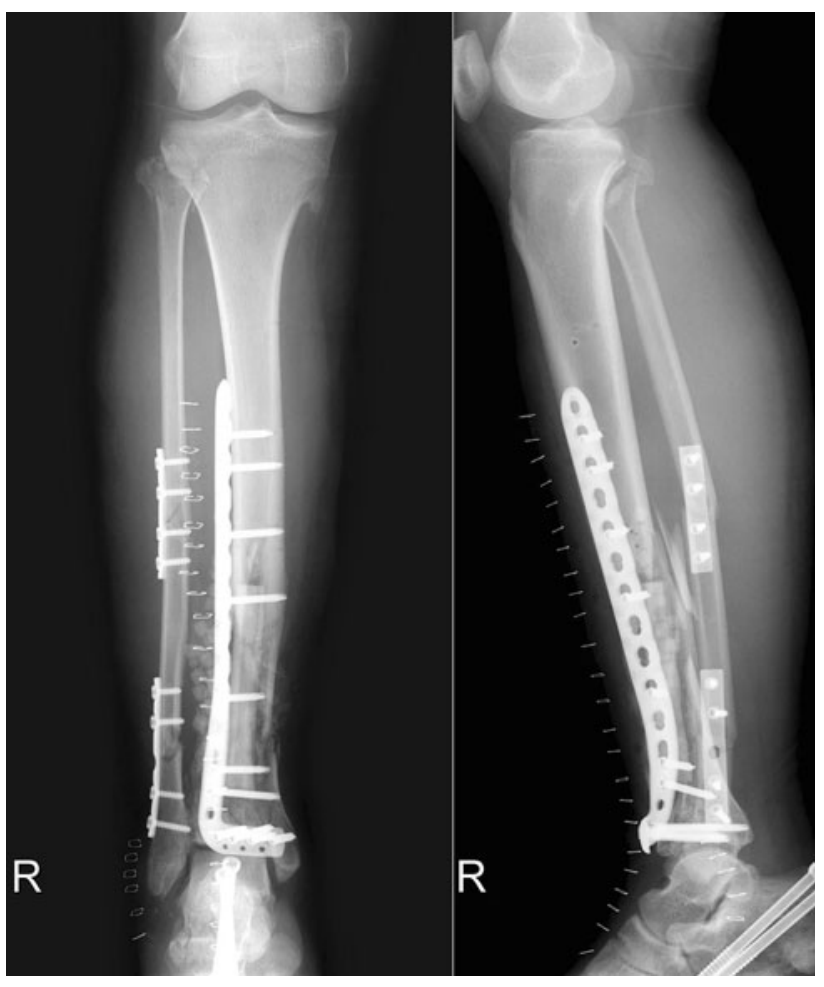

Fig. 4 The pilon fracture treated with a fibular strut allograft and a periarticular locking plate. Postoperative radiograph shows normal alignment and rigid fixation

performed for the pelvis fracture. Closed reduction and nailing was performed for the left femur. An external fixator was temporarily performed for the left humerus and 


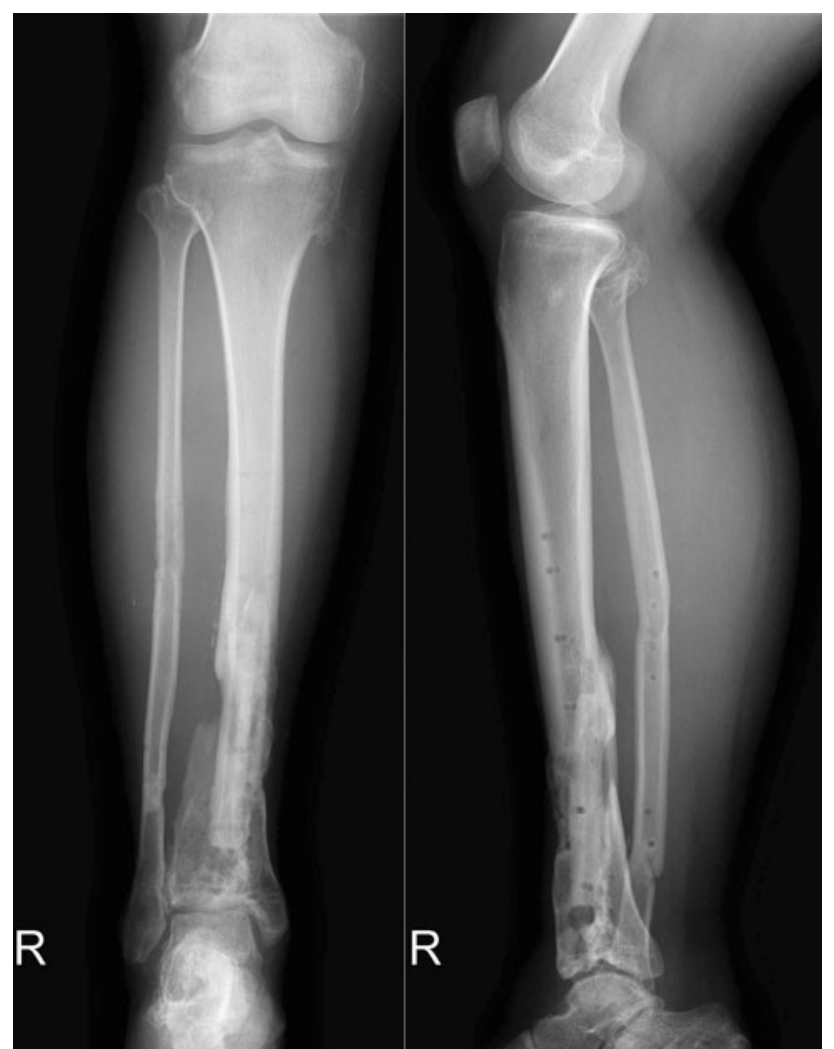

Fig. 5 At the 2-year follow-up, plain radiographs show bone healing and good alignment

both ankle. For the left ankle, irrigation and debridement were performed and then a monofixator was applied temporarily (Fig. 7).

Two weeks later, the open wound was completely healed without infection. The external fixator was removed, and then the bone defect was reconstructed using a fibular strut allograft and ORIF. A fibula strut allograft was inserted in the medullary canal, and a periarticular distal tibia locking plate (Synthes ${ }^{\circledR}$, Switzerland) was applied. A bone substitute was not used, and only an iliac autograft was performed. The patient was also encouraged to do active ankle motion with non-weight bearing. There was no wound problem, and she was able to do partial weight-bearing ambulation at 8 weeks postoperatively, after which the patient was discharged. At the 12-month follow-up, the patient was found to ambulate well without specific problems (Fig. 8).

\section{Discussion}

Because most pilon fractures are caused by high-energy trauma, the pattern of fractures is complex and the adjacent soft tissues are damaged. Therefore, the treatment outcomes are often not satisfactory, and complications such as

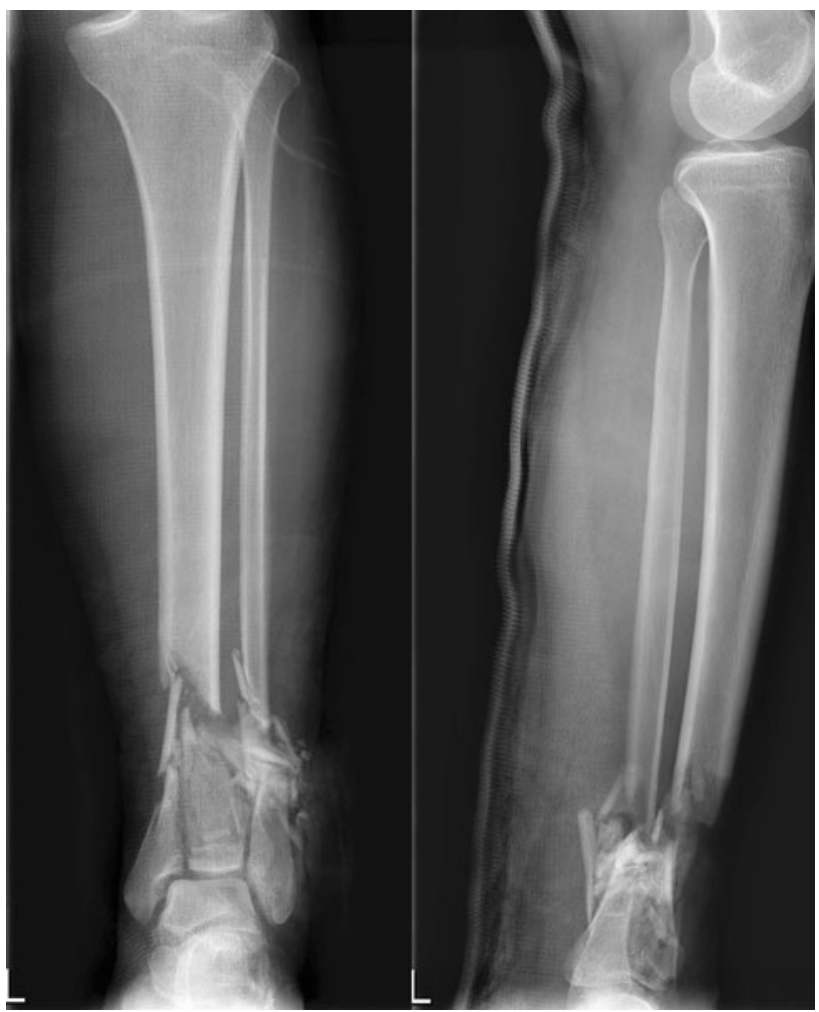

Fig. 6 The plain radiograph shows a highly comminuted pilon fracture with a large bone defect

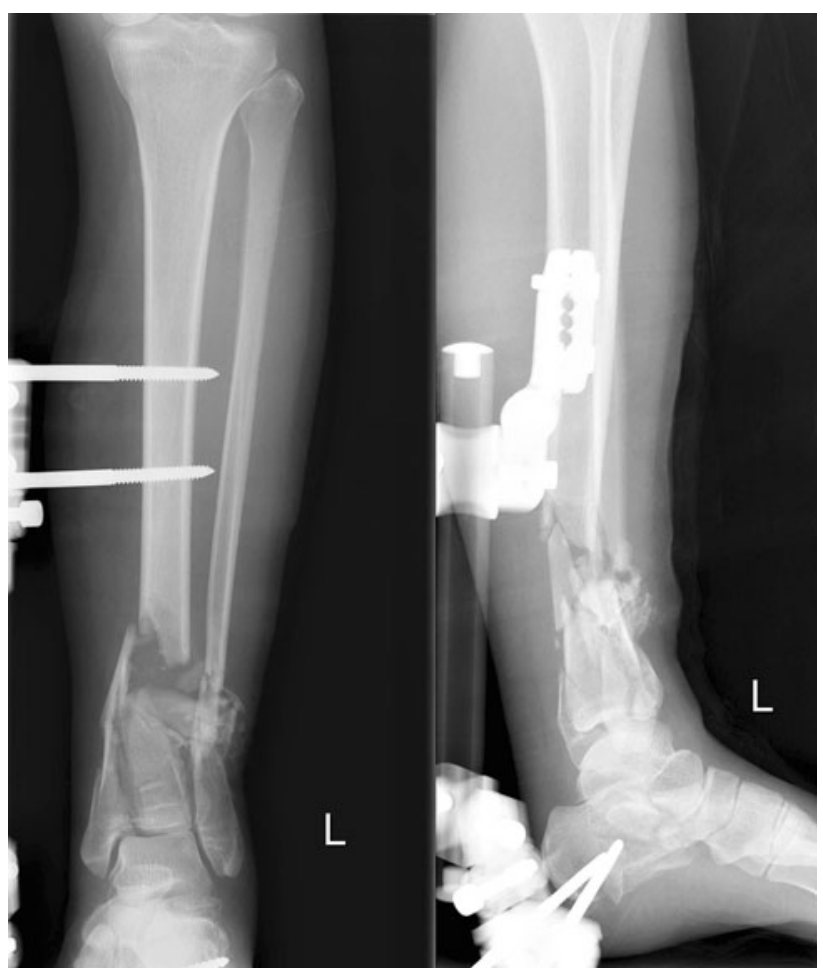

Fig. 7 Application of a spanning external fixator 


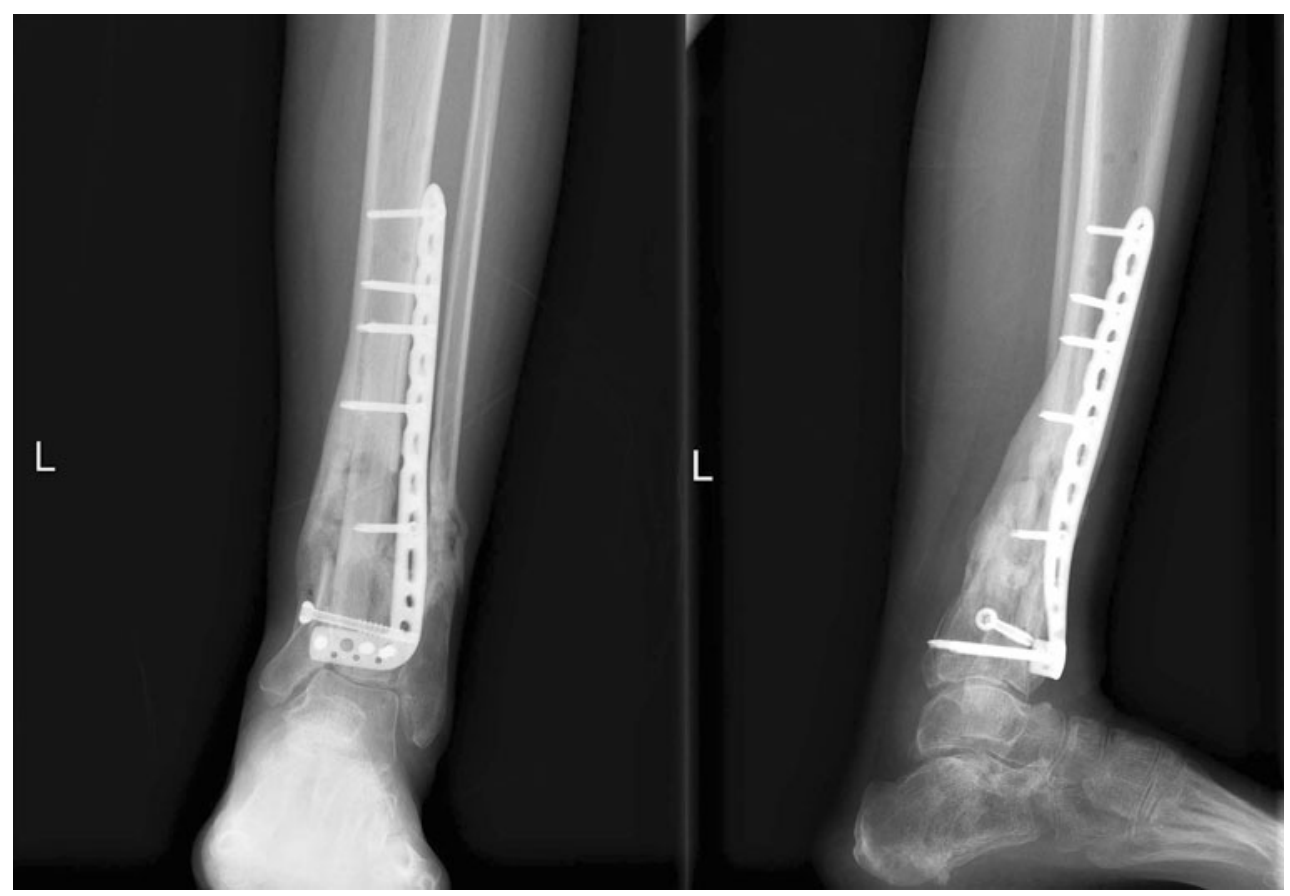

Fig. 8 At the 12-month follow-up, plain radiographs show bone healing and good alignment. The patient was full weight-bearing

infections remain significant $[9,10]$. Also, pilon fractures should obtain accurate reduction due to invading the articular surface. Accordingly, the pilon fracture is difficult to be properly treated. In most cases, pilon fractures are associated with bone defects. In cases of a small-sized defect, the treatment can only be an iliac bone graft. However, the management of pilon fractures with large bone defects is challenging. Several methods have been used to manage these defects. In recent years, methods such as bone grafting, vascularized local or free bone transfer, and distraction osteogenesis have been frequently used. In particular, the free vascularized osteocutaneous fibula graft is helpful for obtaining a sufficient length of the grafted bones [11]. With a synchronous transplantation of soft tissues, such as skin and muscle, one-stage surgery can resolve the defects of soft tissue. There are advantages to these techniques, and satisfactory treatment outcomes can be obtained with them. However, there are disadvantages such as the fatigue fracture of the grafted tibia, complete and incomplete necrosis of a flap, morbidity of the donor site, and functional deficits due to long-term immobilization (e.g., ankle ROM limitation) [11-14]. In addition, most cases of pilon fractures resulted from fall injury are accompanied by fractures in the pelvis and fibula. Thus, we cannot use autogenic iliac bones and fibulas for large segmental bone defects. Therefore, in cases of pilon fractures with large segment bone defects accompanied by multiple fractures, we suggest that the use of fibular strut allograft could be a good treatment of choice.
A structural allograft has previously been used for a bone defect, but it has mainly been used for tumors or for revision surgery of total joint replacements [2]. In 1995, Chmell et al. [2] used a structural allograft for a large segmental defect $>10 \mathrm{~cm}$ in size. But, a structural allograft has been used as an end-stage method for limb salvage of the femur or proximal tibia. As shown in our cases, there were no cases in which initial management was performed for the pilon fracture. Besides, this method does not sacrifice the fibula. We obtained rigid fixation using a periarticular distal tibia locking plate after impaction of the fibular strut allograft. This procedure is effective for patients to perform early articular movement and weight-bearing, and it might be effective for achieving earlier rehabilitation. Also, in our cases, partial weight-bearing ambulation was performed at the 2 nd postoperative month. Approximately 6 months later, almost all normal function was recovered. In particular, the distal tibia is a region in which a great deal of force is concentrated per unit area. Rigid fixation was obtained using a fibular strut allograft, by which the weight-bearing could be done at earlier times. A recent study reported that a long segmental defect was treated with a cylinder mesh cage [15]. However, that defect was in the humerus, which is not a weight-bearing bone. A good result was also reported for the simultaneous treatment of tibia and soft tissue defects with the Ilizarov method [16], but weight-bearing was much delayed in that case.

We aimed for the second-staged surgery with the fibular allograft and ORIF when complete wound healing without 
infection was obtained, because an allograft is very vulnerable to infection; we could treat successfully without infections.

When treating a open pilon fracture in which there is an extension of the fracture to the tibial diaphysis along with a large segmental bone defect $>7 \mathrm{~cm}$ in size and there is the concurrent presence of fibular or pelvic fractures (which rules out bone grafting from these sites), fibular strut allografts and ORIF using periarticular distal tibia locking plates are effective reconstruction options.

Conflict of interest No benefits in any form have been or will be received from a commercial party related directly or indirectly to the subject of this manuscript.

Open Access This article is distributed under the terms of the Creative Commons Attribution Noncommercial License which permits any noncommercial use, distribution, and reproduction in any medium, provided the original author(s) and source are credited.

\section{References}

1. Attias N, Lehmann RE, Bodell LS et al (2005) Surgical management of a long segmental defect of the humerus using a cylindrical titanium mesh cage and plate. A case report. J Orthop Trauma 19:211-216

2. Bishop AT, Wood MB, Sheetz KK (1995) Arthrodesis of the ankle with a free vascularized autogenous bone graft. Reconstruction of segmental loss of bone secondary to osteomyelitis, tumor, or trauma. J Bone Joint Surg Am 77:1867-1875

3. Bone LB (1987) Fractures of the tibial plafond. Orthop Clin North Am 18:95-104

4. Chmell MJ, McAndrew MP, Thomas R et al (1995) Structural allografts for reconstruction of lower extremity open fractures with 10 centimeters or more of acute segmental defects. J Orthop Trauma 9:222-226

5. Cierny G III, Zorn KE (1994) Segmental tibial defects. Comparing conventional and Ilizarov methodologies. Clin Orthop Relat Res 301:118-123

6. Georgiadis GM, De Silva SP (1995) Reconstruction of skeletal defects in the forearm after trauma: treatment with cement spacer and delayed cancellous bone grafting. J Trauma 38:910-914

7. Green SA (1994) Skeletal defects. A comparison of bone grafting and bone transport for segmental skeletal defects. Clin Orthop Relat Res 301:111-117

8. Ilizarov GA, Ledyaev VI (1992) The replacement of long tubular bone defects by lengthening distraction osteotomy of one of the fragments. Clin Orthop Relat Res 280:7-10

9. Lee KS, Park JW (1999) Free vascularized osteocutaneous fibular graft to the tibia. Microsurgery 19:141-147

10. Lindsey RW, Miclau T, Probe R et al (1993) A defect-in-continuity in the canine femur and in vivo experimental model for the study of bone graft incorporation. Yale J Biol Med 66:157-163

11. Low CK, Pho RW, Kour AK et al (1996) Infection of vascularized fibular graft. Clin Orthop Relat Res 323:163-172

12. Mast JW, Teipner WA (1980) A reproducible approach to the internal fixation of adult ankle fractures: Rationale, technique, and early result. Orthop Clin North Am 11:661-679

13. Nonnenmacher J, Bahrm J, Moui Y (1995) The free vascularized fibular transfer as a definitive treatment in femoral septic nonunions. Microsurgery 16:383-387

14. Patel VR, Menon DK, Pool RD et al (2000) Nonunion of the humerus after failure of surgical treatment. Management using the Ilizarov circular fixator. J Bone Joint Surg Br 82:977-983

15. Rozbruch SR, Weitzman AD, Watson JT et al (2006) Simultaneous treatment of tibial bone and soft tissue defect with the ilizarov method. J Orthop Trauma 20:197-205

16. Tu YK, Yen CY, Yeh WL et al (2001) Reconstruction of posttraumatic long bone defect with free vascularized bone graft: good outcome in 48 patients with 6 years follow up. Acta Orthop Scand 72:359-364 